\title{
Effects of a water hammer and cavitation on jet formation in a test tube
}

\author{
Akihito Kiyama $^{1}$, Yoshiyuki Tagawa ${ }^{1} \uparrow$, Keita Ando $^{2}$ and Masaharu Kameda ${ }^{1}$ \\ ${ }^{1}$ Department of Mechanical Systems Engineering, Tokyo University of Agriculture and Technology, \\ Nakacho 2-24-16 Koganei, Tokyo 184-8588, Japan \\ ${ }^{2}$ Department of Mechanical Engineering, Keio University, 3-14-1 Hiyoshi, Kohoku-ku, Yokohama, \\ Kanagawa 223-8522, Japan
}

(Received 11 February 2015; revised 16 November 2015; accepted 18 November 2015; first published online 15 December 2015)

We investigate the motion of a gas-liquid interface in a test tube induced by a large acceleration via impulsive force. We conduct simple experiments in which the tube partially filled with a liquid falls under gravity and hits a rigid floor. A curved gasliquid interface inside the tube reverses and eventually forms a so-called focused jet. In our experiments, there arises either vibration of the interface or an increment in the velocity of the liquid jet, accompanied by the onset of cavitation in the liquid column. These phenomena cannot be explained by a considering pressure impulse in a classical potential flow analysis, which does not account for finite speeds of sound or phase changes. Here we model such water-hammer events as a result of the one-dimensional propagation of a pressure wave and its interaction with boundaries through acoustic impedance mismatching. The method of characteristics is applied to describe pressurewave interactions and the subsequent cavitation. The model proposed is found to be able to capture the time-dependent characteristics of the liquid jet.

Key words: cavitation, interfacial flows (free surface), jets

\section{Introduction}

A liquid jet is of great importance in various industrial and medical processes as well as of fundamental interest as a canonical fluid dynamical phenomenon to study the instability in motion of gas-liquid interfaces (Zeff \& Lathrop 2000; Cooker 2002; Bartolo, Josserand \& Bonn 2006; Bergmann et al. 2008; Duchemin 2008; Eggers \& Villermaux 2008; Tagawa et al. 2013).

A typical liquid jet is one whose tip is sharp and elongated, i.e., the so-called 'focused jet' (Eggers \& Villermaux 2008; Tagawa et al. 2012; Peters et al. 2013). Such a focused jet can be created as follows: a container partially filled with a liquid is instantly set into motion, thus putting all the fluid particles including the gas-liquid interface into rapid acceleration. The accelerated interface then deforms into a focused jet by the flow focusing effect, termed a 'shaped-charge effect' (Birkhoff et al. 1948).

$†$ Email address for correspondence: tagawayo@cc.tuat.ac.jp 
(a)

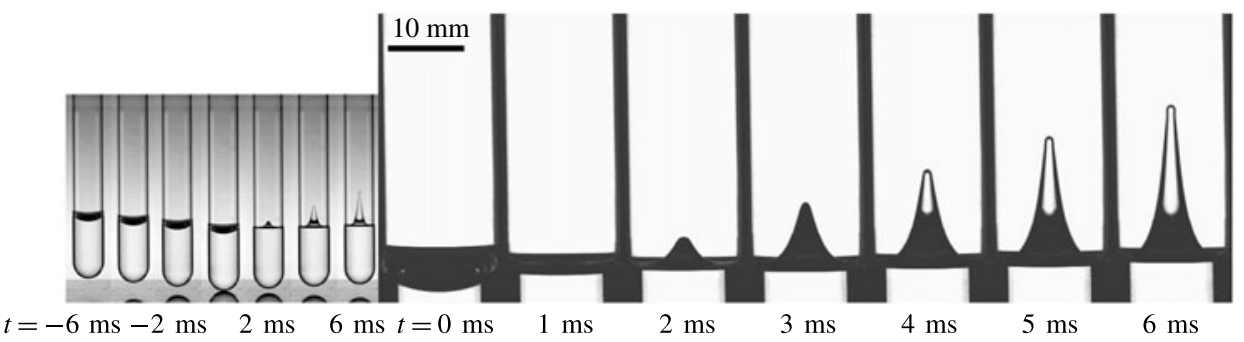

(b)

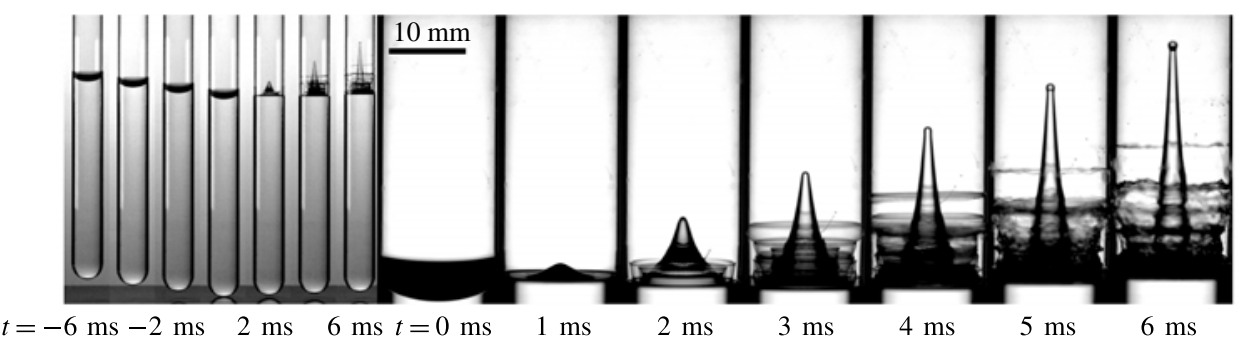

$(c)$

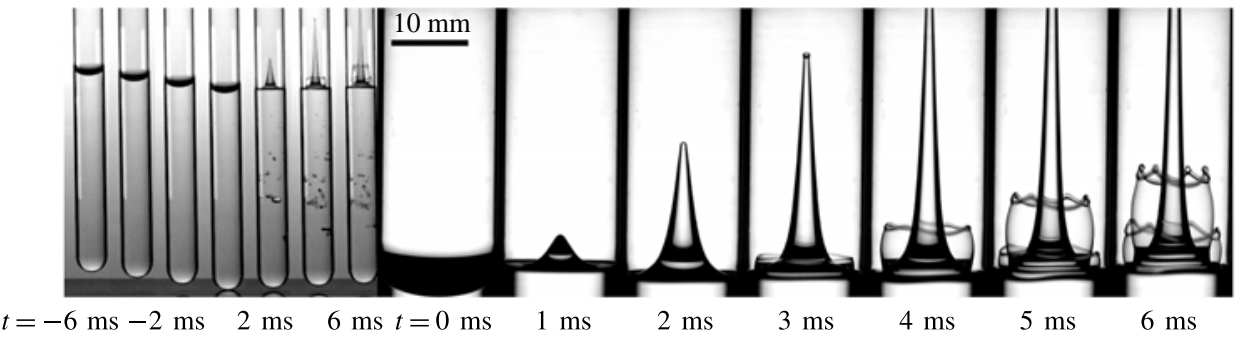

FIgURE 1. Representative examples of observed liquid jets with their classification. (a) Normal type (also see supplementary movie 1). This jet can be described by the (incompressible) pressure impulse. (b) Splash type (supplementary movie 2). Non-trivial vibration of the interface is observed, with small droplets sprayed. (c) Cavitation type (supplementary movie 3). Its jet velocity is much faster than that of the other two types.

A representative example is Pokrovski's experiment (Antkowiak et al. 2007) (see figure 1a, supplementary movie available at http://dx.doi.org/10.1017/jfm.2015.690). In this experiment, a liquid-filled test tube falls freely and eventually collides with a rigid floor. During the tube's free fall (i.e., in the gravity-free state), the shape of the gas-liquid interface quickly becomes hemispherical through the effect of surface tension. Once the tube hits the floor, the direction of its motion reverses. The acceleration of fluids within the tube leads to the formation of a focused jet from the interface.

It is known that a flow triggered by the sudden motion of boundaries can be analysed by considering a pressure impulse (Batchelor 1967; Cooker \& Peregrine 1995) defined as the time integral of pressure evolution. The potential flow theory assumes an instantaneous establishment of pressure fields through the infinite speed of sound, meaning that the characteristic length of acoustic waves is assumed to be much larger than fluid-dynamic length scales to validate the incompressibility condition. Using this pressure impulse approach, Antkowiak et al. (2007) analysed the velocity field right after the impact in Pokrovski's experiment and obtained good agreement with their experiments. Kiyama, Noguchi \& Tagawa (2014) conducted 
similar experiments and found that the jet velocity can be described by the pressure impulse approach as well. The jet velocity can be written as $V_{j}=\alpha U_{0}$, where $U_{0}$ is the velocity of the gas-liquid interface just after the impact (to be explained in detail in $\S 2.1$ ) and $\alpha$ is a dimensionless constant to be determined empirically. The physical meaning of $\alpha$ is the strength of the flow focusing effect after the interface obtains a velocity $U_{0}$.

However, as the impact becomes stronger, we find that the motion of a gas-liquid interface tends to deviate from the previous findings, producing non-trivial vibration of the interface with droplet fragments sprayed (see figure 1(b), supplementary movie 2 ) or an increase in the velocity of a liquid jet accompanied by cavitation in a liquid column (see figure 1(c), supplementary movie 3 ). These phenomena are expected to result from the interaction of compression and expansion waves with boundaries including a gas-liquid interface and the tube walls (Turangan 2013), thereby rendering inappropriate the pressure impulse description based on the potential flow theory.

In this paper, we aim to elucidate the mechanisms of the motions of the gas-liquid interfaces induced by a water hammer as observed in figure $1(b, c)$. For this purpose, we discuss the evolution of the pressure waves as well as the effects of cavitation that may occur in the liquid column. We here propose a one-dimensional wave propagation model based on the method of characteristics and compare it with our experiments.

This paper is organized as follows. In $\S 2$, we show the experimental set-up and observations. We propose a model for describing the observed phenomena in $\S 3$, followed by comparison with experiments in $\S 4$. Section 5 concludes our findings.

\section{Experiment}

\subsection{Experimental set-up and parameters}

Figure 2 shows a schematic diagram of our experimental set-up. We partially fill a test tube with a wetting liquid. The tube is suspended in a test rig by an electromagnet that touches a non-sharp metal piece on the tube's cap. The tube axis is aligned with the vertical axis. When the magnet is turned off, the test tube starts to fall freely from the height $H$ defined as the distance between the bottom of the tube and the floor. The gas-liquid interface is in a gravity-free state and its shape becomes hemispherical before the tube impacts a metal plate on a height-adjustable laboratory jack. We use two high-speed cameras (Photron, Fastcam SA-X) to obtain simultaneously a close-up view of the gas-liquid interface and an overview of the entire liquid column. The frame rate for both cameras is set at 90000 f.p.s., and the exposure time at $8.32 \pm 0.04 \mu \mathrm{s}$. Resolutions are 0.08 and $0.16 \mathrm{~mm} /$ pixel for measuring the motion of the interface and cavitation. Both cameras are triggered by a delay generator (Berkeley Nucleonics, model 575). The tube is illuminated by white-light sources through diffusers. All the equipment is placed on a levelled vibration-isolation table (Newport, Smart Table UT2).

We use (gas-saturated) silicone oil (Sigma Aldrich), whose sonic speed $c_{l}$, density $\rho_{l}$, kinematic viscosity $v$ and vapour pressure $P_{v}$ are respectively $990 \mathrm{~m} \mathrm{~s}^{-1}$, $930 \mathrm{~kg} \mathrm{~m}^{-3}, 10 \mathrm{cSt}$ and $666 \mathrm{~Pa}$ at room temperature. For selected cases, the silicone oil with which the test tube is filled is degassed before the impact experiment to see the effect of dissolved gases on the probability of cavitation. The test tube is made of borosilicate glass whose (longitudinal) sonic speed is about $6000 \mathrm{~m} \mathrm{~s}^{-1}$. The inner diameter $d$ and thickness $h$ of the tube are $14.2 \mathrm{~mm}$ and $1.2 \mathrm{~mm}$, respectively. The bottom of the test tube is rounded, similar to the previous study (Antkowiak et al. 2007). 
(a)

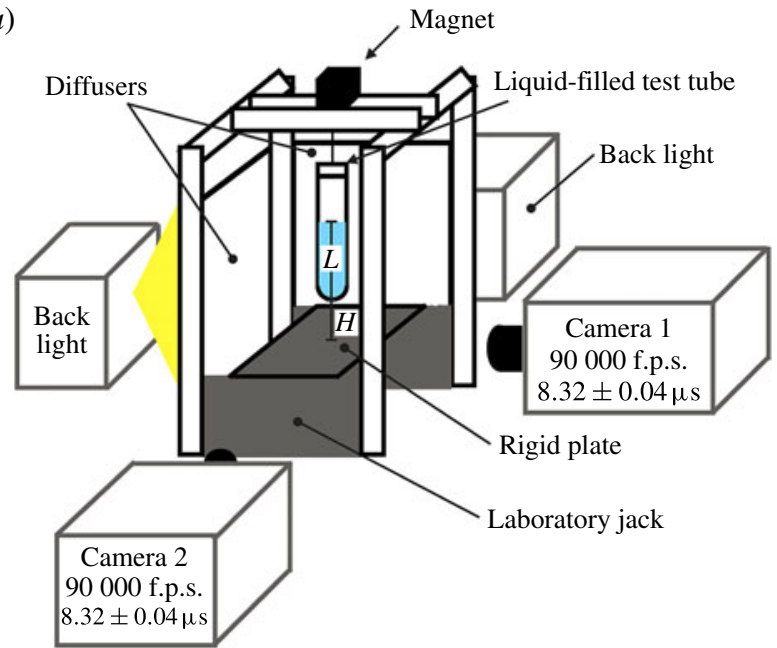

(b)

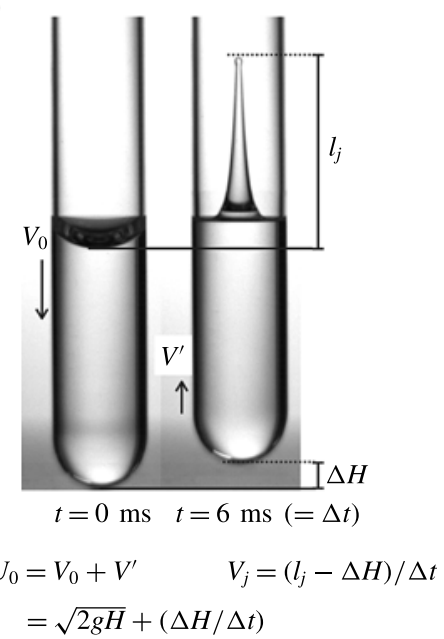

FIGURE 2. (Colour online) Schematic of the experimental set-up and parameters.

For parameter studies, we control the liquid-column height $L$ and the drop height $H$ as shown in figure 2(a). The impact speed $U_{0}$ is defined as the change in the velocity of the liquid column in an inertial frame of reference, $U_{0}=V_{0}+V^{\prime}$, where $V_{0}$ is the speed of the tube relative to the floor just before impact and $V^{\prime}$ is the rebound speed relative to the floor. The speed $V_{0}$ is well approximated by the speed of freely falling bodies $\sqrt{2 g H}$ where $g$ denotes the gravitational acceleration $\left(9.81 \mathrm{~m} \mathrm{~s}^{-2}\right)$. The rebound speed $V^{\prime}$ is calculated as $V^{\prime}=\Delta H / \Delta t$, where $\Delta H$ is the rebound height measured $6 \mathrm{~ms}$ after the impact and $\Delta t=6.0 \mathrm{~ms}$ (see figure $2 b$ ). The jet velocity $V_{j}$ is also calculated in the frame moving with the test tube as $V_{j}=\left(l_{j}-\Delta H\right) / \Delta t$, where $l_{j}$ is the distance of the jet tip from the base of the free surface at impact (see figure $2 b$ ) and $\Delta t=6.0 \mathrm{~ms}$. We repeat the experiment 30 times for each experimental condition.

To classify the phenomena based on whether cavitation occurs in the liquid column, we introduce the cavitation number $K . K$ is a measure of the probability of cavitation: cavitation is more likely to occur as $K$ decreases. It is defined as $K=\left(P_{a t m}-P_{v}\right) / \rho L(a-g)$, where $P_{a t m}, P_{v}, \rho$ and $a$ are respectively the atmospheric pressure, the vapour pressure, the liquid density and the acceleration imposed on the liquid (Daily et al. 2014). The fluid is accelerated upward by the impact, while gravitational acceleration $g$ acts in the opposite direction. Thus, we use $(a-g)$ in the expression for the cavitation number $K$. The acceleration $a$ is measured as $a=U_{0} / \Delta t^{\prime}$, where $U_{0}$ is the impact speed and $\Delta t^{\prime}(=0.5 \mathrm{~ms})$ is the duration of the collision between the tube and the floor (Young 1989), which is estimated from high-speed camera images.

We judge that cavitation occurs if bubbles larger than 1 pixel $(=0.16 \mathrm{~mm})$ are detected in the captured image. The threshold of $K<1$ results in approximately $60 \%$ of visually detected cavitation bubbles in gas-saturated silicone oil in our experiment.

\subsection{Preliminary experiment}

To determine the parameters in the main experiment (in §2.3) where we explore the effects of a water hammer and cavitation on jet formation, we first perform a preliminary experiment using gas-saturated silicone oil and make a rough sketch 


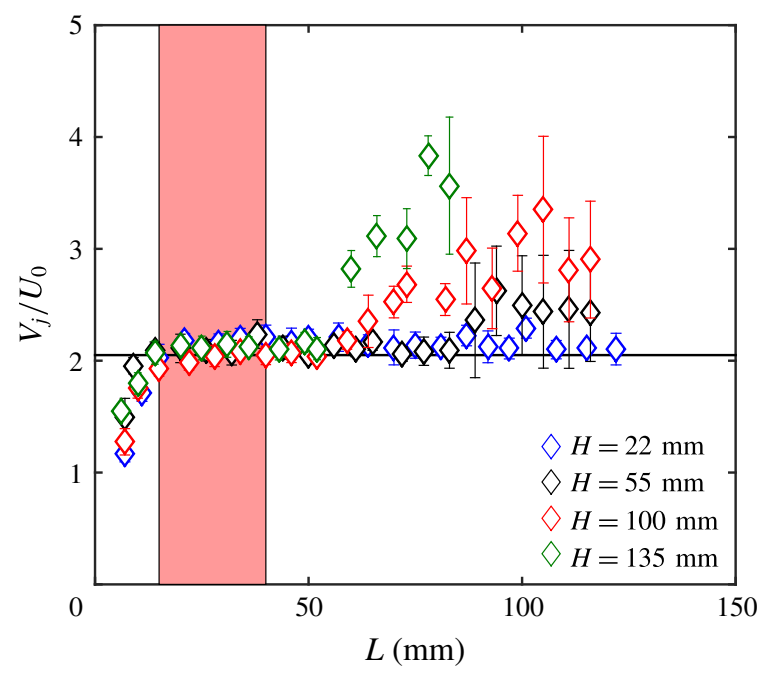

FIgURE 3. (Colour online) Preliminary experiment on the jet velocity $V_{j}$ for selected drop heights $H$ with varying liquid-column height $L$.

regarding the relation between the jet velocity $V_{j}$ and the impact velocity $U_{0}$. The experiment is performed over broad ranges of the drop height $H(22,55$, $100,135 \mathrm{~mm}$ ) and the liquid-column height $L$ (from 5 to $120 \mathrm{~mm}$ ). We repeat measurements more than five times in each experimental condition. The results are summarized in figure 3. Markers and error bars in the figure represent mean values and their standard deviations, respectively.

For $L<15 \mathrm{~mm}$, the jet velocity is smaller than data in the highlighted area due to the shallow depth of the liquid.

For $15 \mathrm{~mm} \leqslant L \leqslant 40 \mathrm{~mm}$ (highlighted area), cavitation never occurs and a plateau regime of $V_{j} / U_{0}$ exists. The jet velocity can be described as $V_{j}=\alpha U_{0}$, indicating that the flow can be described by the potential theory (or the pressure impulse approach). Recall that $\alpha$ corresponds to the strength of the flow focusing effect and is calculated as $\alpha=2.05 \pm 0.25$ in this regime, where the mean value and standard deviation are estimated from data for $15 \mathrm{~mm} \leqslant L \leqslant 40 \mathrm{~mm}$.

For $L>40 \mathrm{~mm}$, the jet velocity is increased in the case where cavitation exists (figure 1c). In this paper, we mainly discuss the phenomena (figure $1 b, c$ ) in this regime.

\subsection{Main experiment}

Based on the preliminary test described in $\$ 2.2$, we conduct a series of experiments as summarized in table 1 . In this main experiment, we measure the jet velocity $V_{j}$ with varying liquid height $L$, mainly using gas-saturated silicone oil. We characterize jet formation based on the jet shape and the occurrence of cavitation inside the liquid column. To be specific, we categorize all the jets into three types: 'normal-type', 'splash-type' and 'cavitation-type'. In the case of $K=1.02$ (see figure $1 a$ and supplementary movie 1), we obtain a normal-type jet as observed in previous experiments (Antkowiak et al. 2007; Kiyama et al. 2014) in which cavitation is not detected. On the other hand, for $K<1$ jet shapes are apparently different from the normal-type jet. Unless cavitation occurs, there arise a non-trivial vibration of the 


\begin{tabular}{|c|c|c|c|c|c|c|c|}
\hline $\begin{array}{l}L \\
(\mathrm{~mm})\end{array}$ & $\begin{array}{c}H \\
(\mathrm{~mm})\end{array}$ & $\begin{array}{c}U_{0} \\
\left(\mathrm{~m} \mathrm{~s}^{-1}\right)\end{array}$ & $\begin{array}{c}h \\
(\mathrm{~mm})\end{array}$ & $\begin{array}{l}M \\
(\mathrm{~g})\end{array}$ & $\begin{array}{c}K \\
(-)\end{array}$ & Degassed & $\begin{array}{c}\text { Cavitation } \\
\text { probability }(\%)\end{array}$ \\
\hline 15 & 73 & $1.73 \pm 0.02$ & 1.2 & 26.9 & $2.11 \pm 0.01$ & No & 0 \\
\hline 20 & 73 & $1.74 \pm 0.02$ & 1.2 & 26.9 & $1.56 \pm 0.01$ & No & 0 \\
\hline 25 & 73 & $1.77 \pm 0.02$ & 1.2 & 26.9 & $1.24 \pm 0.01$ & No & 0 \\
\hline 30 & 73 & $1.79 \pm 0.02$ & 1.2 & 26.9 & $1.02 \pm 0.01$ & No & 0 \\
\hline 45 & 73 & $1.78 \pm 0.02$ & 1.2 & 26.9 & $0.68 \pm 0.01$ & No & 43.3 \\
\hline 60 & 73 & $1.72 \pm 0.02$ & 1.2 & 26.9 & $0.53 \pm 0.01$ & No & 100 \\
\hline 60 & 73 & $1.72 \pm 0.02$ & 1.2 & 26.9 & $0.53 \pm 0.01$ & Yes & 6.7 \\
\hline 75 & 73 & $1.66 \pm 0.02$ & 1.2 & 26.9 & $0.44 \pm 0.01$ & No & 30.0 \\
\hline 90 & 73 & $1.56 \pm 0.02$ & 1.2 & 26.9 & $0.39 \pm 0.01$ & No & 60.0 \\
\hline
\end{tabular}

TABLE 1. Parameters for the main experiment: the liquid-column height $L$, the drop height $H$, the impact velocity $U_{0}$, the thickness of the tube $h$, the mass of the tube $M$, the cavitation number $K$, and whether the liquid is degassed. The experiment is repeated 30 times for each condition.

interface and the formation of small droplets (figure $1 b$ and supplementary movie 2); we call this a splash-type jet. Once cavitation occurs, even for the same value of $K$ (but possibly with a different state of cavitation nuclei), in contrast, the jet becomes much faster than jets of the other two types (figure $1 c$ and supplementary movie 3); we call this a cavitation-type jet. Moreover, spray formation is not obtained in the cavitation type. Throughout this paper, the jets we observed are grouped into these types.

Since the state of cavitation nuclei including their size and location cannot be controlled in our experiments, the appearance of cavitation we detected optically is a random event (Mørch 2015). In the case of the cavitation-type jet, bubbles prefer to appear in the neighbourhood of the bottom of the tube, where the largest tension is expected to be achieved; the probability of having bubbles near the bottom of the tube is roughly $80 \%$ out of all the cavitation-type events. In the remaining events (about $20 \%$ ), on the other hand, bubbles appear in the middle of the liquid column (as seen in figure $1 c$ ).

Figure 4(a) presents the temporal evolution of the jet velocities for both splash and cavitation types. The measured velocity is averaged over $\pm 0.1 \mathrm{~ms}$ to smooth out its fluctuation. For both cases, the jet velocity reaches its maximum at $t \approx 2-3 \mathrm{~ms}$ and subsequently shows a gradual decline. We note, for the cavitation type, that cavitation bubbles inside the liquid column collapse at $t \approx 2 \mathrm{~ms}$ as inferred by image analysis. Clearly, a deviation in the jet velocity between the two cases appears just after the jet formation $(t \approx 1 \mathrm{~ms})$.

Figure $4(b)$ compares the jet velocities for each type. The vertical axis is the normalized jet velocity $V_{j} /\left(\alpha U_{0}\right)$, where $\alpha=2.05$ based on the preliminary experiment. The jet velocity of the splash type is less than 1.2 times faster than the normal type for all $L$. In contrast, the jet velocity of the cavitation type for $L=90 \mathrm{~mm}$ is 1.5 times faster.

It is instructive to note that the probability of cavitation can be reduced by degassing liquids (Milgram 1969). To confirm this effect, we repeat the experiment for $L=60 \mathrm{~mm}$ and $H=73 \mathrm{~mm}$ in degassed silicone oil. As expected, the probability of cavitation is reduced from $100 \%$ to $6.7 \%$ so that the velocity increment in the cavitation-assisted jet is not obtained. 

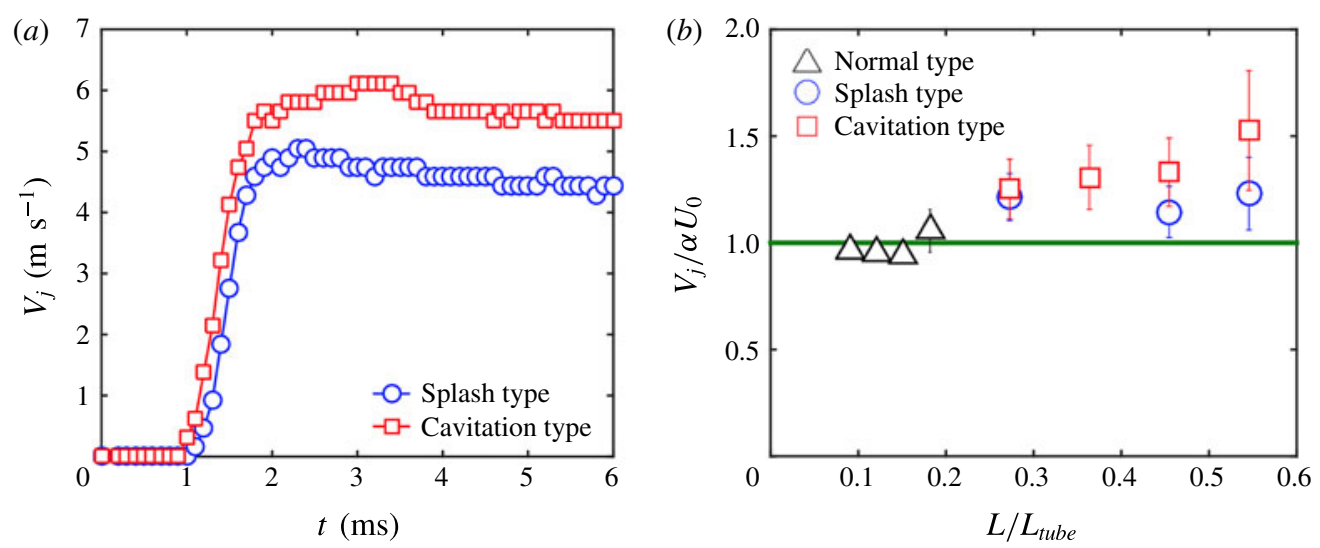

FIgURE 4. (Colour online) (a) Temporal evolution of the jet velocities for splash and cavitation types for liquid height $L=90 \mathrm{~mm}$; $(b)$ the jet velocity versus the liquid height.

\section{Model}

The unsteady features of the splash- and cavitation-type jets ( $\$ 2$ ) cannot be explained by potential flow analysis with the incompressibility constraint. Thus what we discuss here is the phenomena within a few periods of acoustic oscillations $4 L / c_{l} \sim O(0.1) \mathrm{ms}$, which is short compared to the focusing that occurs on a fluid-flow time scale of $d / 2 V_{j} \sim O(1) \mathrm{ms}$. The aim is to provide the velocity of the gas-liquid interface $U$ after a few acoustic time scales, during which acoustic waves keep being trapped inside the column through reflections at boundaries. The flow focusing effect then follows in the fluid-dynamic time scales, leading to the jet velocity $V_{j}=\alpha U$.

For clarity and simplicity, we adopt a one-dimensional plane wave model, ignoring the curvature of capillarity and the bottom of the tube with following assumptions: (i) the cross-sectional area of the tube is constant throughout the direction of propagation; (ii) the tube wall is rigid, (iii) acoustic waves are linear, (iv) the medium is inviscid unless cavitation occurs. The extent of fluid-structure interaction may be quantified by the dimensionless parameter $\beta=\left(c_{l}^{2} / c_{s}^{2}\right)\left(\rho_{l} / \rho_{s}\right)(d+h) / h$ where $c$ is the speed of longitudinal sound and subscripts $l$ and $s$ denote liquid and solid phases, respectively (Shepherd \& Inaba 2010). In this particular example, we have $\beta \approx 0.1<1$, indicating that tube deformation is expected to be small. The effects of structural oscillation and the inertia of the tube will be investigated based on supporting experiment in $\S 4$. The pressure perturbations of tens of megapascals are in the range of validity of the theory of linear acoustics (Thompson 1972). As a result of assumptions (ii) and (iii), acoustic waves in the tube are anticipated to propagate at the speed of sound in the liquid.

Linear wave interactions at boundaries separating different materials can be modelled by the acoustic relation that can be derived from the linearized laws of mass and momentum conservation. Wave reflections can be quantified based on the acoustic impedance, which is a thermodynamic property defined as $I=\rho c$, the product of density $\rho$ and the speed of (longitudinal) sound $c$; the acoustic impedances of gas, liquid and solid phases are denoted by $I_{g}, I_{l}$ and $I_{s}$, respectively. In the extreme cases where an incident wave travels from very stiff to soft materials (e.g., liquid to gas; $I_{l} \gg I_{g}$ ) and vice versa (e.g., liquid to solid; $I_{l} \ll I_{s}$ ), the acoustic relation becomes very simple. If a wave in liquid collides with a gas-liquid interface, the 
(a)

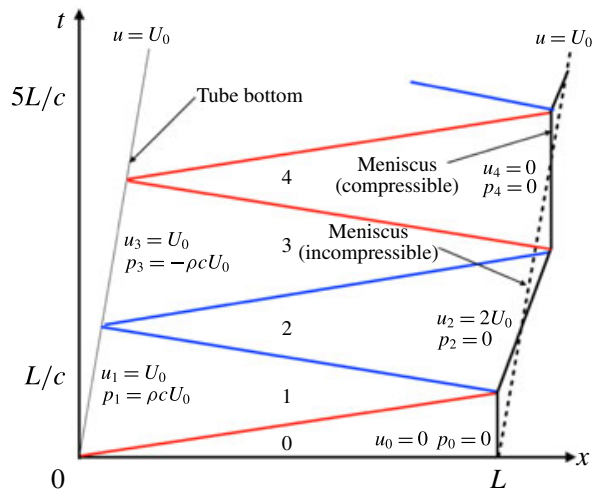

(b)

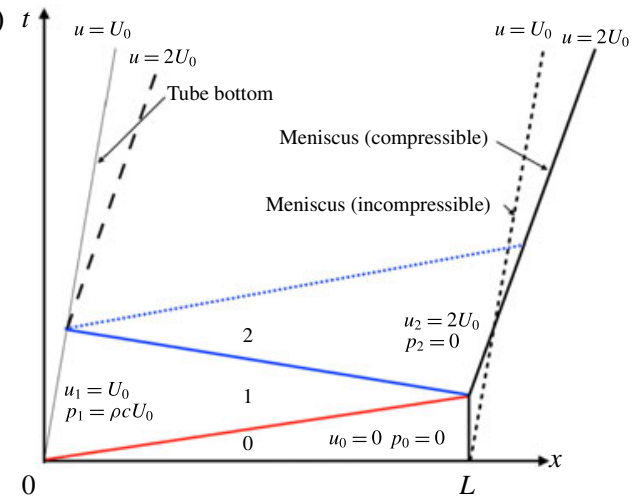

FIGURE 5. (Colour online) $x-t$ diagrams for $(a)$ splash-type jet and $(b)$ cavitation-type jet. The red and blue solid lines refer to compression and expansion waves, respectively. The thin black line and the bold black line show the positions of the bottom of the tube and the gas-liquid interface, respectively.

wave transmission to the gas is so small that pressure at the interface remains almost undisturbed (i.e., a free boundary). In this case, the interfacial velocity becomes twice the particle velocity induced by the incident wave. In contrast, even when a wave in a liquid collides with a solid boundary, the boundary is essentially fixed (i.e., a rigid boundary) and the resulting pressure doubles as a result of superposition of the incident and reflected waves. In the present experiments, the acoustic impedances of the gas, liquid and solid phases are calculated, respectively, as $I_{g}=4.0 \times 10^{2} \mathrm{~Pa} \mathrm{~s} \mathrm{~m}^{-1}$, $I_{l}=9.3 \times 10^{5} \mathrm{~Pa} \mathrm{~s} \mathrm{~m}^{-1}$ and $I_{s}=1.2 \times 10^{7} \mathrm{~Pa} \mathrm{~s} \mathrm{~m}^{-1}$.

With the acoustic relations in these extreme cases, we draw $x-t$ diagrams of acoustic wave propagation based on the method of characteristics for jets of normal, splash and cavitation types in figure 5. The diagram starts at the moment when the tube wall is set into motion with velocity $U_{0}$. According to assumption (iv), wave attenuation due to dissipative effects is not considered.

First we explain how waves evolve in the normal and splash types. As mentioned in $\$ 2.2$, jet formation for the normal type can be described by a pressure impulse in the incompressible sense whereby the pressure field is increased instantaneously in the entire flow of concern. Thus, the evolution of the gas-liquid interface starts to move at $U=U_{0}$ (see the bold dashed line in figure $5 a$ ). Recall that the velocity $U$ here is not the jet velocity $V_{j}$, but the velocity of the free surface which has not yet been accelerated by the kinematic flow focusing.

For splash-type jets, on the other hand, a pressure wave propagates at the speed of sound in the liquid and is trapped within the liquid column through multiple reflections; see the red and blue lines in figure 5(a) that denote compression and expansion waves, respectively. The induced velocities of the liquid at states 0 to 4 in the diagram are $u_{0}=0, u_{1}=U_{0}, u_{2}=2 U_{0}, u_{3}=U_{0}$ and $u_{4}=0$, respectively. This results in (periodic) vibration of the gas-liquid interface between $U=0$ and $U=2 U_{0}$. This means that the free surface evolves at the average velocity $U_{0}$ (the same as in the normal jet) but with fluctuation $\pm U_{0}$ through multiple wave reflections. The frequency of the interface vibration $f_{l}$ for the case of $L=90 \mathrm{~mm}$ and $c=990 \mathrm{~m} \mathrm{~s}^{-1}$ is approximated by $c / 4 L \sim 2.8 \mathrm{kHz}$.

Next, we model cavitation induced by wave interaction in the cavitation-type jet (see figure $5 b$ ). Cavitation is expected to occur if liquid pressure is below a threshold 
value (e.g., vapour pressure if one ignores surface tension and the dynamics of nuclei bubbles of heterogeneous cavitation). The compression wave initially generated at the bottom of the tube reaches the gas-liquid interface and is reflected as an expansion wave. The velocity of the gas-liquid interface at this moment is $U=2 U_{0}$. The expansion wave then reaches the bottom of the tube and is reflected as an expansion wave; unless cavitation occurs, negative pressure in the gauge is obtained in the liquid after the expansion wave passes by (i.e., state 3 in figure $5 a$ ). This means that the liquid is stretched and its pressure can possibly be below the cavitation threshold if the initial impact is sufficiently large. If cavitation occurs soon after the passage of the expansion wave, the pressure in state 3 in figure $5(a)$ will be relaxed towards the vapour pressure (or one atmosphere if air dissolved in the liquid turns into cavitation bubbles). If the expansion wave is significantly damped, the velocity of the gas-liquid interface $U$ is expected to be undisturbed at $2 U_{0}$. Here, the attenuation of the expansion wave may be assumed to be proportional to the work done in the creation of cavitation bubbles or simply to the volume of the bubbles. In this sense, the velocity of the gas-liquid interface $U$ could be correlated to the volumes of cavitation bubble. We introduce an empirical formula to estimate the velocities of cavitation-type jets:

$$
U=U_{0}+C \Omega /(S \tau),
$$

where $C$ is a dimensionless fitting constant, $\Omega$ is the maximum volume of cavitation bubbles, $\tau$ is time for bubble growth and $S$ is the cross-sectional area of the tube $\left(S=\pi d^{2} / 4\right)$.

The maximum volume of cavitation bubbles $\Omega$ is inferred by image analysis. We treat each bubble as a binarized spot. We estimate the centre of gravity for each bubble, then measure the mean distance in vertical direction between the top/bottom points and the centre as the typical radius of the bubble. We take the error as \pm 1 pixel $(=0.16 \mathrm{~mm})$ for bubble radii.

Finally, it is instructive to note that the cavitation modelling in figure $5(b)$ is based on the assumption that cavitation occurs instantly at $t=2 L / c_{l}$ and thus appears from the bottom of the tube that is first exposed to tension. As explained in $\S 2.3$, bubbles were more likely to appear from the bottom of the tube, which is consistent with our model assumption. In the other cases, bubbles appeared in the middle of the column, giving rise to a time lag in the relaxation of pressure to the vapour pressure. However, the time lag in such an acoustic event is so short (compared with the fluid-dynamic jet formation) that where cavitation bubbles appear in the liquid column is expected to be less important.

\section{Comparison}

We now re-examine the experimental data on the basis of the wave propagation analysis as well as the empirical formula (3.1) for the cavitation type developed in $\S 3$.

First, we show a frequency analysis of meniscus motion in the neighbourhood of the gas-liquid contact line for each type in figure 6. For a splash-type jet in figure $6(b)$, there is a strong peak around $3.1 \mathrm{kHz}$, which is not observed in the other two types. This indicates multiple reflections of pressure waves trapped between the gas-liquid interface and the bottom of the tube (see figure 5a). The observed frequency of $3.1 \mathrm{kHz}$ is found to be close to the frequency of the acoustic waves in the liquid of $2.8 \mathrm{kHz}$ from $f_{l}=c / 4 L$. The height of the tube's rounded bottom is approximately $9 \mathrm{~mm}$ and the radius of curvature of the meniscus just before impact is approximately $7 \mathrm{~mm}$. Thus the net length of the liquid column for wave 

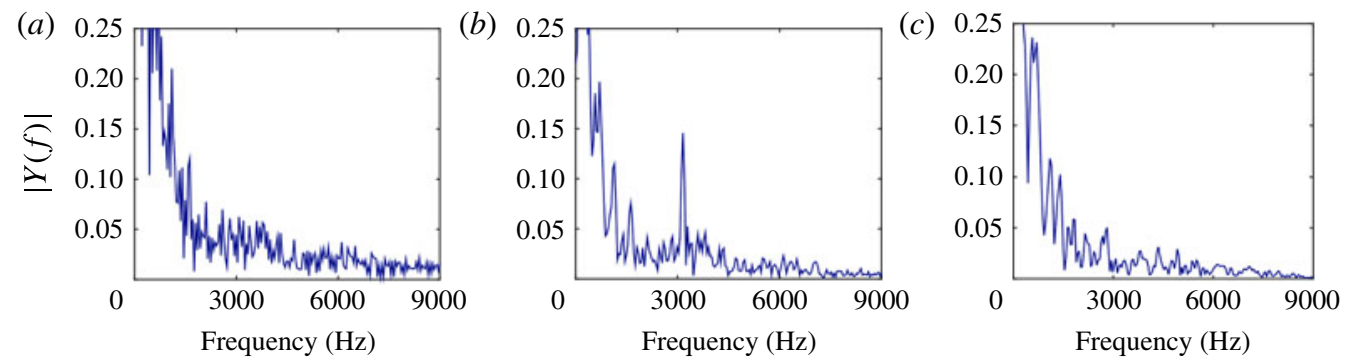

FIGURE 6. (Colour online) Frequency analysis of meniscus motion in the neighbourhood of the contact line for $(a)$ normal-type jet, $(b)$ splash-type jet and $(c)$ cavitation-type jet.

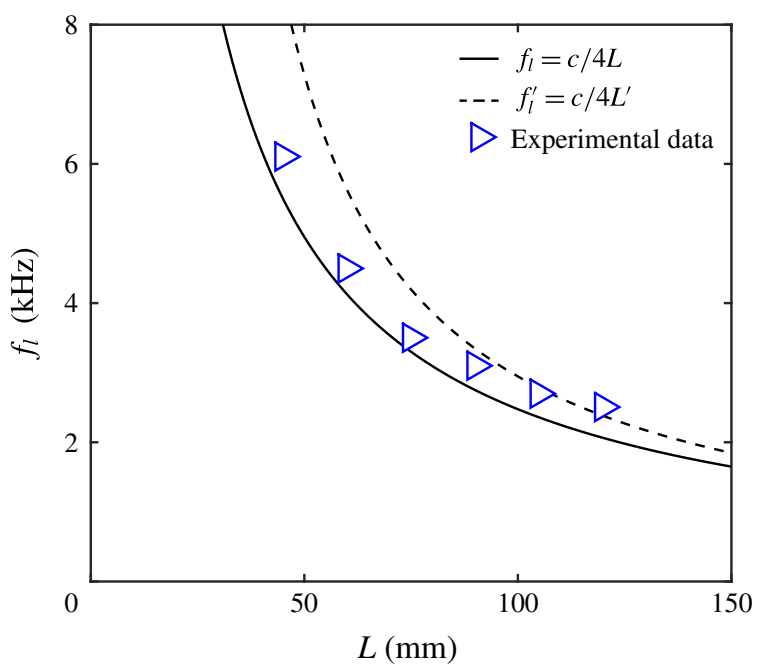

FIGURE 7. (Colour online) The vibration frequency of the meniscus $f_{l}$ versus the liquid depth $L$.

propagation $L^{\prime}$ may be $\sim 74 \mathrm{~mm}$ instead of $L=90 \mathrm{~mm}$, which results in a slightly larger frequency than the predicted value $2.8 \mathrm{kHz}$. Thus, lower and upper bounds of the predicted frequency of the free surface may be defined, respectively, as $f_{l}^{\prime}=c_{l} / 4 L^{\prime}$ and $f_{l}=c_{l} / 4 L$. Such a peak does not exist in the cavitation-type jet in figure $5(c)$, indicating that the liquid pressure is effectively relaxed to the bubble pressure, which acoustically hinders wave transmission from the liquid phase.

To further support the proposed scenario based on wave reflection within the liquid column, we examine the non-trivial vibration of the free surface for a splash-type jet with varying liquid-column height $L$ from 45 to $120 \mathrm{~mm}$ (see the experimental condition in the supplementary material). Figure 7 shows the observed frequency together with the lower and upper bounds of the predicted frequency. Most of the observed values of frequency can be found between the two bounds. As expected, the frequency is inversely proportional to the liquid-column height $L$. This suggests that the vibration indeed arises from repeated wave reflection within the column. Furthermore, we examine the non-trivial vibration of the interface with varying thickness of the tube $h$ from 2.0 to $3.2 \mathrm{~mm}$ (hence the mass of the tube $M$ from 42.7 to $74.5 \mathrm{~g}$ ) in order to investigate the effects of the tubes. We choose 


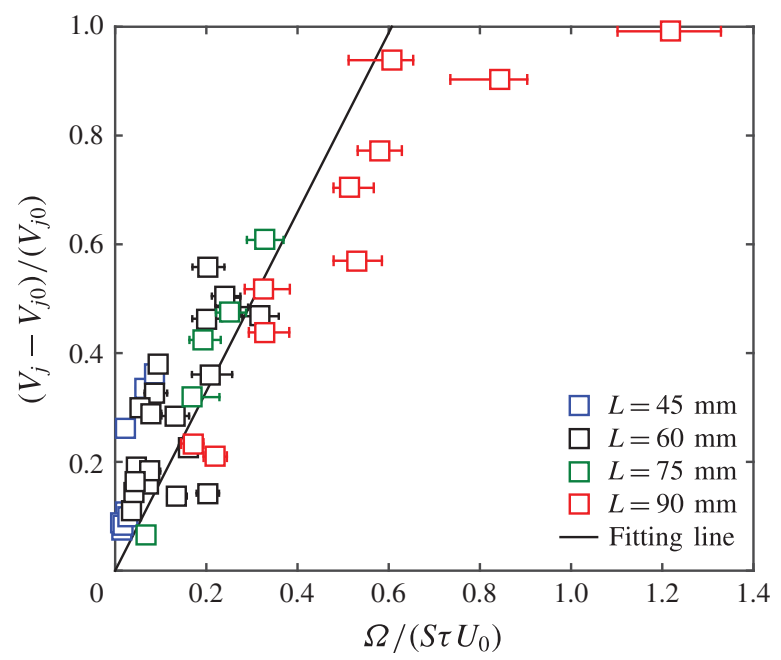

FIgURE 8. (Colour online) The velocity of the cavitation-type jet as a function of the volume of cavitation bubbles.

the drop height $H=73 \mathrm{~mm}$ and the liquid-column height $L=90 \mathrm{~mm}$. The detailed experimental condition is shown in the supplementary material. It is confirmed that the vibration frequency of the free surface is measured again as $3.1 \mathrm{kHz}$ even though the tube thickness $h$ is varied and the natural frequency of oscillations in the tube's deformation is thus altered. This suggests that the observed vibration of the free surface is rather insensitive to the structural oscillations and is governed mainly by the acoustic wave interaction.

Second, we compare the empirical formula (3.1) to the measured velocity of cavitation-type jets in figure 8 . The vertical and horizontal axes show, respectively, the jet velocity increment $\left(V_{j}-V_{j_{0}}\right) / V_{j_{0}}$ where $V_{j 0}=\alpha U_{0}$ and the bubble volume $\Omega /\left(S \tau U_{0}\right)$. We use a different colour for each liquid height $L$ in figure 8 . The data for all $L$ collapse into a single band, implying that the jet velocity increases as the bubble volume increases and is rather insensitive to where cavitation bubbles are nucleated. The measurements are fitted to the empirical formula (3.1). In this fitting, we exclude the data for $\left(V_{j}-V_{j 0}\right) / V_{j 0}>0.7$ that show a significant deviation from the asymptotic behaviour of the empirical formula; the fitting constant $C$ is calculated as 1.2 (see the solid line in figure 8). It is interesting to note that all the velocity increments $\left(V_{j}-V_{j_{0}}\right) / V_{j_{0}}$ do not go beyond unity. This may also support our model, which predicts a maximum velocity increment up to unity (see figure $5(b)$ and $\S 3$ ).

Finally, we summarize how acoustic effects deteriorate the classical pressure impulse approach. If pressure waves induced by the impact are strong enough to trigger acoustic phenomena such as non-trivial vibration or cavitation, the classical approach no longer holds and we recommend that the acoustic model we have proposed be applied.

\section{Conclusion}

In this paper, we conducted water-hammer experiments in which a test tube partially filled with a liquid falls under gravity and hits a rigid floor. We found new types of a jet formed at the gas-liquid interface whose unsteady features cannot be captured by 
the classical potential flow theory. We categorized liquid jets into three types (normal, splash and cavitation types) based on jet shape and the occurrence of cavitation inside a liquid column (see figure 1). The splash-type jets showed continuous vibration of the gas-liquid interface while the other two types did not. The velocities of cavitation-type jets were found to be much greater than those of the other two types and accompanied by the onset of cavitation inside the liquid.

In order to understand the phenomena, we proposed a new model to explain the propagation of the pressure wave and its interaction with the boundaries and cavitation. For splash-type jets, the vibration of the interface was caused by repeated wave reflection within the liquid column. Thus the vibration frequency can be estimated from the liquid-column height and the speed of sound. For cavitation-type jets, we considered the attenuation of expansion waves due to pressure relaxation around cavitation bubbles, which leads to the emergence of a faster liquid jet. We speculated that the velocity of the cavitation-type jet can be correlated to the displaced volume of bubbles (3.1). We compared the vibration frequency of the gas-liquid interface for a splash-type jet and found a reasonable agreement between experiments and the model (see figure 6). We also compared the empirical formula (3.1) with the experiments and found that the increase in velocity for the cavitation-type jet can be well estimated from the displaced volume of cavitation bubbles (figure 8).

\section{Acknowledgements}

We thank Y. Watanabe, M. Maeshima and K. Hirose for helping with our experiments. This work was supported by JSPS KAKENHI grant number 26709007.

\section{Supplementary movies}

Supplementary movies are available at http://dx.doi.org/10.1017/jfm.2015.690.

\section{REFERENCES}

Antkowiak, A., Bremond, N., Le Dizès, S. \& Villermaux, E. 2007 Short-term dynamics of a density interface following an impact. J. Fluid Mech. 577, 241-250.

Bartolo, D., Josserand, C. \& Bonn, D. 2006 Singular jets and bubbles in drop impact. Phys. Rev. Lett. 96 (12), 124501.

Batchelor, G. K. 1967 An Introduction to Fluid Dynamics. Cambridge University Press.

Bergmann, R., De Jong, E., Choimet, J. B., Van Der Meer, D. \& Lohse, D. 2008 The origin of the tubular jet. J. Fluid Mech. 600, 19-43.

Birkhoff, G., MacDougall, D. P., Pugh, E. M. \& Taylor, G. I. 1948 Explosives with lined cavities. J. Appl. Phys. 19 (6), 563-582.

CoOKer, M. J. 2002 Unsteady pressure fields which precede the launch of free-surface liquid jets. Proc. R. Soc. Lond. A 458, 473-488.

Cooker, M. J. \& Peregrine, D. H. 1995 Pressure-impulse theory for liquid impact problems. J. Fluid Mech. 297, 193-214.

Daily, J., Pendlebury, J., Langley, K., Hurd, R., Thomson, S. \& Truscott, T. 2014 Catastrophic cracking courtesy of quiescent cavitation. Phys. Fluids 26 (9), 091107.

Duchemin, L. 2008 Self-focusing of thin liquid jets. Proc. R. Soc. Lond. A 464 (2089), 197-206.

Eggers, J. \& Villermaux, E. 2008 Physics of liquid jets. Rep. Prog. Phys. 71 (3), 036601.

KiYama, A., Noguchi, Y.\& TAgawa, Y. 2014 The generation of a liquid jet induced by a pressure impulse (experimental study on the jet velocity). Trans. JSME 80 (814), FE0151.

Milgram, J. H. 1969 The motion of a fluid in a cylindrical container with a free surface following vertical impact. J. Fluid. Mech. 37 (3), 435-448. 
Mørch, K. A. 2015 Cavitation inception from bubble nuclei. Interface Focus 5, 20150006.

Peters, I. R., Tagawa, Y., Oudalov, N., Sun, C., Prosperetti, A., Lohse, D. \& van der MeER, D. 2013 Highly focused supersonic microjets: numerical simulations. J. Fluid Mech. 719, 587-605.

ShePherd, J. E. \& INABA, K. 2010 Shock loading and failure of fluid-filled tubular structure. In Dynamic Failure of Materials and Structures, chap. 6, pp. 153-190. Springer.

Tagawa, Y., Oudalov, N., El Ghalbzouri, A., Sun, C. \& Lohse, D. 2013 Needle-free injection into skin and soft matter with highly focused microjets. Lab. on a Chip 13 (7), 1357-1363.

Tagawa, Y., Oudalov, N., Visser, C. W., Peters, I. R., van der Meer, D., Sun, C., Prosperetti, A. \& Lohse, D. 2012 Highly focused supersonic microjets. Phys. Rev. X 2 (3), 031002.

Thompson, P. A. 1972 Compressible-Fluid Dynamics. McGraw-Hill.

Turangan, C. K. 2013 Highly focused microjet simulations using the free-Lagrange method (FLM). In ICMF2013: Proc. 8th Int. Conf. Multiphase Flow, Korea, vol. 232, pp. 1-8.

Young, F. R. 1989 Cavitation. McGraw-Hill.

Zeff, B. W., Kleber, B., Fineberg, J. \& Lathrop, D. P. 2000 Singularity dynamics in curvature collapse and jet eruption on a fluid surface. Nature 403, 401-404. 